\title{
Edge states and determination of pairing symmetry in superconducting $\mathrm{Sr}_{2} \mathrm{RuO}_{4}$
}

\author{
K. Sengupta, Hyok-Jon Kwon, and Victor M. Yakovenko \\ Department of Physics and Center for Superconductivity Research, University of Maryland, College Park, MD 20742-4111 \\ cond-mat/0106198, v.1: 11 June 2001; v.2: 2 September; v.3: 20 November; v.4: 11 January 2002)
}

\begin{abstract}
We calculate the energy dispersion of the surface Andreev states and their contribution to tunneling conductance for the order parameters with horizontal and vertical lines of nodes proposed for superconducting $\mathrm{Sr}_{2} \mathrm{RuO}_{4}$. For vertical lines, we find double peaks in tunneling spectra reflecting the van Hove singularities in the density of surface states originating from the turning points in their energy dispersion. For horizontal lines, we find a single cusp-like peak at zero bias, which agrees very well with the experimental data on tunneling in $\mathrm{Sr}_{2} \mathrm{RuO}_{4}$.
\end{abstract}

PACS numbers: 74.70.Pq, 74.80.Fp, 73.20.-r

\section{INTRODUCTION}

Numerous experiments suggest that the superconducting state of $\mathrm{Sr}_{2} \mathrm{RuO}_{4}$ [1] is unconventional (see review (2)). Strong suppression of $T_{c}$ by non-magnetic impurities [3] and absence of the Hebel-Slichter peak in NMR experiments [- indicate that it is not $s$-wave. The absence of spin susceptibility reduction below $T_{c}$ in the Knight shift measurements indicates the spin-triplet pairing [5]. The $\mu \mathrm{SR}$ experiment suggests a superconducting state with broken time-reversal symmetry [6]. Small-angle neutron scattering reveals a square vortex lattice, which is interpreted as the indication for a two-component order parameter [7]. These experiments initially led to suggestion of the $2 \mathrm{D}$ chiral (time-reversal non-invariant), isotropic, nodeless $p$-wave pairing potential [8]. However, the power-law temperature dependences found in specific heat [9], nuclear relaxation rate [10], penetration depth 11], thermal conductivity [12], and ultrasonic attenuation 13.14] indicate nodes in the energy gap. In response to these experiments, the following alternative order parameters were proposed [15]: anisotropic $p$-wave [16], $p$-wave with horizontal lines of nodes [17, and $f$ waves with vertical [17,18] or horizontal lines of nodes [19]. It was also proposed that the $\alpha$ and $\beta$ bands of $\mathrm{Sr}_{2} \mathrm{RuO}_{4}$ are either not superconducting 20] or have horizontal lines of nodes 21]. Big in-plane anisotropy of ultrasound attenuation [14] may support the vertical lines of nodes. However, thermal conductivity depends very little on the orientation of an in-plane magnetic field [22], which is against the vertical lines 23].

Electron tunneling between a normal metal and a superconductor proved to be an important tool in determining superconducting symmetry. Observation of the zerobias conductance peak (ZBCP) 24] due to the formation of the midgap Andreev bound states [25] confirmed the $d$-wave symmetry of the high- $T_{c}$ cuprates. Electron tunneling into a three-dimensional $p$-wave superconductor with the pairing potential corresponding to the B-phase of ${ }^{3} \mathrm{He}$ was considered in Ref. [26]. Electron tunneling in $\mathrm{Sr}_{2} \mathrm{RuO}_{4}$ was studied theoretically in Refs. 27] and 28] for the $2 \mathrm{D}$ isotropic chiral unitary and non-unitary $p$ waves. In this paper, we calculate the tunneling conductance curves for the alternative order parameters listed above and compare them with experiments [29,30].

\section{GENERAL FORMALISM}

We model the tunneling contact by two semi-infinite regions, normal $(\mathrm{N})$ and superconducting $(\mathrm{S})$, with a flat interface (I) perpendicular to the $a$ axis of $\mathrm{Sr}_{2} \mathrm{RuO}_{4}$ (see Fig. 1). The $x$ and $y$ axes are selected along the crystal axes $a$ and $b$, respectively. The tunneling conductance is calculated at zero temperature by solving the Bogoliubov-de Gennes equations in the ballistic regime following Refs. [31, 32]. The tunneling barrier is modeled by a delta-function potential of strength $\mathcal{H}$, so the boundary conditions at the interface are $\left.\psi_{n}\right|_{I}=\left.\psi_{s}\right|_{I}$ and $\left.\hat{v}_{n} \psi_{n}\right|_{I}=\left.\hat{v}_{s} \psi_{s}\right|_{I}-\left.2 i \mathcal{H} \psi_{n}\right|_{I}$. Here $\psi_{n}$ and $\psi_{s}$ are the electron wave functions in the normal and superconducting regions, and $\hat{v}_{n, s}$ are the velocity components perpendicular to the interface. The pairing potential of a triplet superconductor can be expressed as $\hat{\Delta}=i \hat{\sigma}_{y}(\hat{\boldsymbol{\sigma}} \cdot \mathbf{d}) \Delta(\mathbf{k}, x)$, where $\hat{\boldsymbol{\sigma}}$ are the Pauli matrices operating on the electron spin indices, the vector $\mathbf{d}$ indicates the direction of spin polarization, and $\mathbf{k}$ is the relative momentum of electrons in a Cooper pair. In this paper, we consider the cases where $\mathbf{d}$ does not depend on $\mathbf{k}$ and is pinned to the $c$ axis of $\mathrm{Sr}_{2} \mathrm{RuO}_{4}$. The spatial dependence of the pairing potential is taken to be step-like: $\Delta(\mathbf{k}, x)=\Delta(\mathbf{k}) \Theta(x)$. Assuming that the electron momentum $k_{y}$ parallel to the interface is conserved, the tunneling conductance per one layer, $\bar{G}(V)$, can be written as an integral over $k_{y}$ 31, 32]:

$$
\begin{aligned}
\bar{G}(V) & =\frac{2 e^{2}}{h} l \int_{-k_{y}^{\max }}^{k_{y}^{\max }} \frac{d k_{y}}{h} G\left(V, k_{y}\right), \\
G\left(V, k_{y}\right) & =D \frac{1+|\Gamma|^{2} D-(1-D)|\Gamma|^{4}}{\left|1-(1-D) \Gamma^{2} \exp (i \Phi)\right|^{2}} .
\end{aligned}
$$

In Eq. (1), $e$ is the electron charge, $h$ is the Planck constant, $l$ is the length of the interface, and $V$ is the 


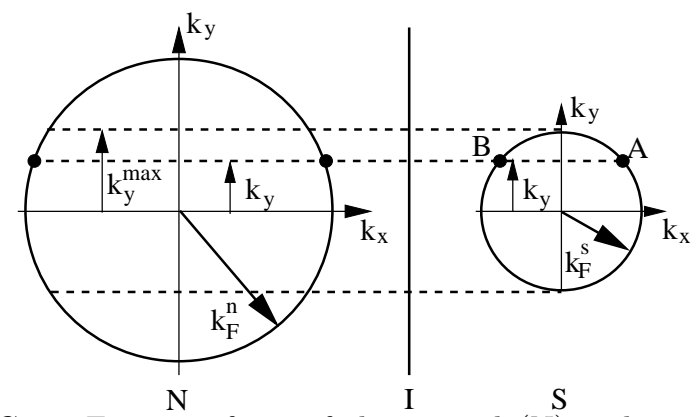

FIG. 1. Fermi surfaces of the normal (N) and superconducting $(\mathrm{S})$ materials forming the interface I. The points A and $\mathrm{B}$ on the Fermi surface $\mathrm{S}$ are connected by specular reflection from the interface.

bias voltage. We consider the case where the Fermi surfaces of both the normal metal and superconductor are circular, and their radii are $k_{F}^{(n)}>k_{F}^{(s)}$, as shown in Fig. 1. The limits of integration are set by the smaller Fermi momentum: $k_{y}^{\max }=k_{F}^{(s)}$. In Eq. (2), $D\left(k_{y}\right)=4 v_{n}\left(k_{y}\right) v_{s}\left(k_{y}\right) /\left\{\left[v_{n}\left(k_{y}\right)+v_{s}\left(k_{y}\right)\right]^{2}+4 \mathcal{H}^{2}\right\}$ is the normal-state transmission coefficient, where $v_{n, s}\left(k_{y}\right)$ are the Fermi velocities components perpendicular to the interface. $\Phi\left(k_{y}\right)=\phi_{A}\left(k_{y}\right)-\phi_{B}\left(k_{y}\right)$ is the phase difference of the superconducting pairing potentials at the points on the Fermi surface connected by electron reflection from the interface (points A and B in Fig. 1) [32]. $\Gamma\left(V, k_{y}\right)$ is

$$
\begin{aligned}
& \Gamma=\frac{e V-\operatorname{sgn}(e V) \sqrt{(e V)^{2}-\left|\Delta\left(k_{y}\right)\right|^{2}}}{\left|\Delta\left(k_{y}\right)\right|}, \quad|e V| \geq|\Delta|, \\
& \Gamma=\frac{e V-i \sqrt{\left|\Delta\left(k_{y}\right)\right|^{2}-(e V)^{2}}}{\left|\Delta\left(k_{y}\right)\right|}, \quad|e V| \leq|\Delta| .
\end{aligned}
$$

Denoting $G\left(V, k_{y}\right)$ as $\mathcal{G}\left(V, k_{y}\right)$ for the subgap voltage $|e V| \leq|\Delta|$, where $|\Gamma(V)|=1$, we rewrite Eq. (2) as

$$
\begin{aligned}
& \mathcal{G}\left(V, k_{y}\right)=\frac{D^{2}\left(k_{y}\right) / 2\left[1-D\left(k_{y}\right)\right]}{D^{2}\left(k_{y}\right) / 4\left[1-D\left(k_{y}\right)\right]+F^{2}\left(V, k_{y}\right)}, \\
& F=\sqrt{1-|e V / \Delta|^{2}} \cos (\Phi / 2)-(e V /|\Delta|) \sin (\Phi / 2) .
\end{aligned}
$$

For a high barrier, $D \approx v_{n} v_{s} / \mathcal{H}^{2} \ll 1$. Then Eq. (4) becomes $\mathcal{G}\left(V, k_{y}\right) \approx \pi D\left(k_{y}\right) \delta\left[F\left(V, k_{y}\right)\right]$, and Eq. (1) gives

$$
\overline{\mathcal{G}}(V) \approx C \int_{-k_{y}^{\max }}^{k_{y}^{\max }} d k_{y} v_{n}\left(k_{y}\right) v_{s}\left(k_{y}\right) \delta\left[F\left(V, k_{y}\right)\right],
$$

where $C=2 \pi e^{2} l / h^{2} \mathcal{H}^{2}$. The delta-function contributes to the integral (6) when $F\left(V, k_{y}\right)=0$. Using Eq. ([5), this condition can be written as $e V=E\left(k_{y}\right)$, where

$$
E\left(k_{y}\right)=\left|\Delta\left(k_{y}\right)\right| \cos \left[\Phi\left(k_{y}\right) / 2\right]
$$

for $\sin (\Phi / 2) \geq 0$, i.e. $0 \leq \Phi \leq 2 \pi$, which is the appropriate interval for $\Phi . E\left(k_{y}\right)(\overline{7})$ is nothing but the energy of a surface Andreev state with the momentum $k_{y}$ obtained for the impenetrable barrier $(\mathcal{H} \rightarrow \infty)$ [25]. The energy density of these surface states (DOS) is

$$
\rho(\epsilon)=\int_{-k_{F}^{(s)}}^{k_{F}^{(s)}} \frac{d k_{y}}{h} \delta\left[\epsilon-E\left(k_{y}\right)\right]=\sum_{j} \frac{1}{h\left|\partial_{k_{y}^{(j)}} E\left(k_{y}^{(j)}\right)\right|},
$$

where $k_{y}^{(j)}$ is the $j$-th root of the equation $E\left(k_{y}\right)=\epsilon$.

The delta-function in Eq. (6) indicates that the subgap tunneling takes place when the bias voltage matches the energy of a surface state: $e V=E\left(k_{y}\right)$. Denoting the $j$-th root of this equation as $k_{y}^{(j)}$ and resolving the deltafunction in Eq. (6), we find

$\overline{\mathcal{G}}(V) \approx C \sum_{j} \frac{v_{n}\left(k_{y}^{(j)}\right) v_{s}\left(k_{y}^{(j)}\right) \sqrt{\left|\Delta\left(k_{y}^{(j)}\right)\right|^{2}-(e V)^{2}}}{\left|\partial_{k_{y}^{(j)}} E\left(k_{y}^{(j)}\right)\right|}$.

Because the denominators in Eqs. (9) and (8) are the same, both DOS and tunneling conductance exhibit peaks (the inverse-square-root van Hove singularities) at the turning points of the energy dispersion, where $\partial_{k_{y}} E\left(k_{y}\right)=0$. The peak positions depend solely on the pairing potential $\Delta\left(k_{y}\right)$ through Eq. (7) and not on the band structure details. The number of such turning points does not change upon small continuous deformation of $\Delta\left(k_{y}\right)$, so it is a topological feature. However, because of the additional factors in Eq. (9), tunneling conductance is not simply proportional to DOS, as often assumed. Eq. (9) vanishes at $k_{y} \rightarrow k_{F}^{(s)}$, where the normal component $v_{s}$ of the Fermi velocity goes to zero. It also vanishes when $\Delta\left(k_{y}\right) \rightarrow 0$ or $E\left(k_{y}\right) \rightarrow \pm \Delta\left(k_{y}\right)$ (i.e. $\left.\Phi\left(k_{y}\right) \rightarrow 0,2 \pi\right)$. In these cases, the quasiparticle localization length perpendicular to the interface, $\lambda=\hbar v_{s} / \sqrt{|\Delta|^{2}-E^{2}}$, diverges [25]; thus, the probability to find a quasiparticle on the surface vanishes.

\section{APPLICATION TO $\mathrm{Sr}_{2} \mathrm{RuO}_{4}$}

In Figs. 2, 3, 田, and 月, we show how this general formalism applies to the alternative superconducting order parameters proposed for $\mathrm{Sr}_{2} \mathrm{RuO}_{4}$ in the literature. In order to focus on the features of superconductor, rather than normal metal, we took $k_{F}^{(n)}=5 k_{F}^{(s)}$. Then, the perpendicular velocity of the normal metal is approximately constant for all momenta $\left|k_{y}\right| \leq k_{F}^{(s)}: v_{n} \approx v_{F}^{(n)}$, while in the superconductor it is $v_{s}=v_{F}^{(s)} \sqrt{1-\left(k_{y} / k_{F}^{(s)}\right)^{2}}$, where $v_{F}^{(n)}$ and $v_{F}^{(s)}$ are the corresponding Fermi velocities. The numerical calculations are done for the high barrier $Z=\mathcal{H} / \sqrt{v_{F}^{(n)} v_{F}^{(s)}}=3$. As the figures illustrate, in this limit, the approximate curves given by Eq. (9), which includes only the contribution of the localized surface states, agree well with the exact curves obtained from Eqs. (1) and (2), which also include the contribution of the extended bulk states. 


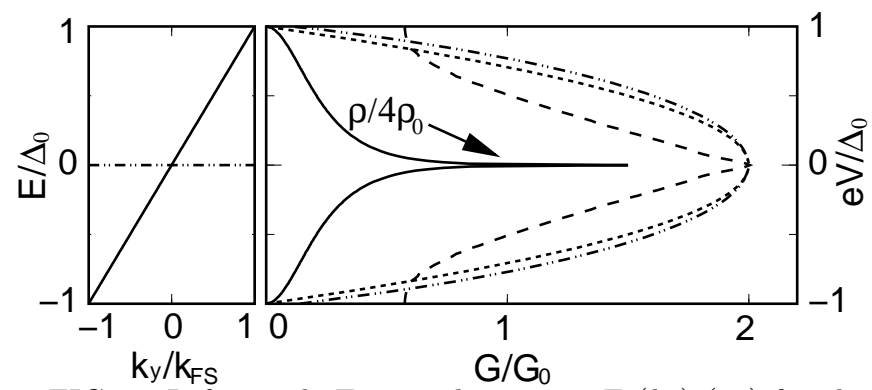

FIG. 2. Left panel: Energy dispersion $E_{1}\left(k_{y}\right)$ (10) for the $2 \mathrm{D}$ isotropic $p$-wave. Right panel: The corresponding exact [Eqs. (17) and (2), dash-dotted line] and approximate [Eq. (12), dotted line] tunneling conductance curves; DOS (solid line) and tunneling conductance (long-dashed line) for the $p$-wave with horizontal lines of nodes [Eq. (19)].

\section{A. Nodeless pairing potential}

For the 2D isotropic chiral $p$-wave, the pairing potential and the energy dispersion of the edge states are [8,28]

$$
\Delta_{1}(\mathbf{k})=\Delta_{0}\left(k_{x}+i k_{y}\right) / k_{F}^{(s)}, \quad E_{1}\left(k_{y}\right)=\Delta_{0} k_{y} / k_{F}^{(s)} .
$$

Substituting Eq. (10) into Eqs. (8) and (9), we find

$$
\begin{aligned}
& \rho(\epsilon)=k_{F}^{(s)} / h \Delta_{0} \equiv \rho_{0}, \\
& \overline{\mathcal{G}}(V) \approx 2 G_{0}\left[1-\left(e V / \Delta_{0}\right)^{2}\right],
\end{aligned}
$$

where $G_{0}=\pi e^{2} l k_{F}^{(s)} v_{F}^{(n)} v_{F}^{(s)} / h^{2} \mathcal{H}^{2}$ is the normal-state conductance. The energy dispersion $E_{1}\left(k_{y}\right)(10)$ has no turning points, and the corresponding DOS (11) is flat: $\rho(\epsilon)=$ const. The tunneling conductance curve $\bar{G}(V)$ (12) is parabolic for $|e V|<\Delta_{0}$, attaining the maximal value $2 G_{0}$ at $V=0$ and vanishing at $|e V|=\Delta_{0}$, as shown in Fig. 2. For $|e V|>\Delta_{0}, \bar{G}(V)$ increases with increasing $|V|$ and saturates at the normal-state value $G_{0}$ at $|e V| \gg \Delta_{0}$. This behavior is in agreement with previous numerical calculations [27,28]. One can recognize that the curves shown in Fig. 4 of Ref. [28] and the corresponding figures of Ref. [27] for $|e V|<\Delta_{0}$ are actually distorted parabolas. The deviations are due to the finite values of $Z$ and to the finite size of the acceptance cone utilized in the numerical calculations [27,28]. Our analytical expression (12) applies in the case $Z \gg 1$ and for the full acceptance cone.

\section{B. Pairing potentials with vertical lines of nodes}

In this subsection, we consider pairing potentials with vertical lines of nodes, which originate from modulation of the gap function $\Delta(\mathbf{k})$ in the $\left(k_{x}, k_{y}\right)$ plane.

For the anisotropic chiral $p$-wave proposed in Ref. [16], the pairing potential and the energy dispersion are

$$
\begin{aligned}
\Delta_{2}(\mathbf{k}) & =\Delta_{0}\left[\sin \left(k_{x} a / \hbar\right)+i \sin \left(k_{y} a / \hbar\right)\right], \\
E_{2}\left(k_{y}\right) & =\Delta_{0} \sin \left(k_{y} a / \hbar\right),
\end{aligned}
$$

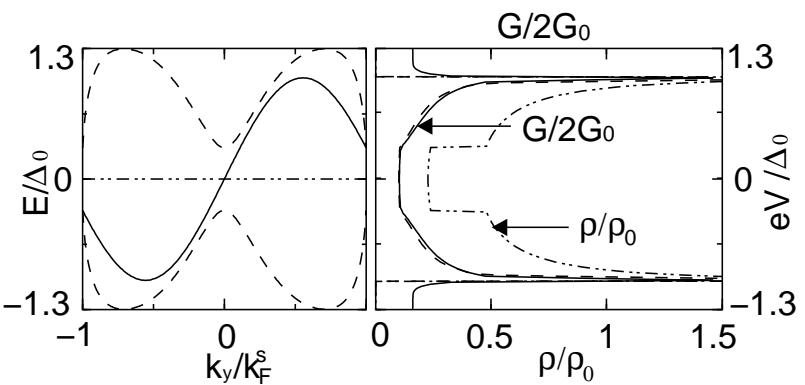

FIG. 3. Left panel: Energy dispersion $E_{2}\left(k_{y}\right)$ [Eq. (14), solid line] and gap $\left|\Delta_{2}\left(k_{y}\right)\right|$ [Eq. (13), dashed line] for the anisotropic $p$-wave. Right panel: the corresponding DOS [dashed-dotted line] and the exact (solid line) and approximate [dashed line] tunneling conductance curves.

where $a$ is the lattice constant, and $k_{F}^{(s)} a=0.9 \pi \hbar$. Substituting Eqs. (13) and (14) into Eqs. (11), (2), (3), (9) and (8), we calculate the tunneling conductance and DOS curves shown in Fig. 3. They exhibit peaks at $e V= \pm \Delta_{0}$ originating from the turning points in the edge states dispersion. Equation $e V=E\left(k_{y}\right)$ has one solution $k_{y}^{(j)}$ for $|e V|<0.31 \Delta_{0}$ and two solutions for $0.31 \Delta_{0}<|e V|<\Delta_{0}$. The switch causes the discontinuity of DOS and the slope change of tunneling conductance at $e V= \pm 0.31 \Delta_{0}$.

For the $f$-wave proposed in Ref. [17, we have

$$
\begin{aligned}
\Delta_{3}(\mathbf{k}) & =\Delta_{0}\left(k_{x}^{2}-k_{y}^{2}\right)\left(k_{x}+i k_{y}\right) /\left(k_{F}^{(s)}\right)^{3}, \\
E_{3}\left(k_{y}\right) & =\Delta_{0}\left|k_{x}^{2}-k_{y}^{2}\right| k_{y} /\left(k_{F}^{(s)}\right)^{3} .
\end{aligned}
$$

As shown in Fig. 1, the edge states dispersion has cusps at $k_{y}= \pm k_{F}^{(s)} / \sqrt{2}$, where $\Delta_{3}(\mathbf{k})$ vanishes, and two turning points in between. Consequently, the tunneling conductance and DOS curves have peaks at $\mathrm{eV}= \pm 0.26 \Delta_{0}$.

For another $f$-wave proposed in Refs. 17, 18], we have

$$
\begin{aligned}
\Delta_{4}(\mathbf{k}) & =2 \Delta_{0} k_{x} k_{y}\left(k_{x}+i k_{y}\right) /\left(k_{F}^{(s)}\right)^{3}, \\
E_{4}\left(k_{y}\right) & =2 \Delta_{0} k_{x}^{2} k_{y} /\left(k_{F}^{(s)}\right)^{3} .
\end{aligned}
$$

Eqs. (17) and (18) transform into Eqs. (15) and (16) upon a $\pi / 4$ rotation in the $x-y$ plane. As shown in Fig. 5 , the turning points in the energy dispersion and the peaks

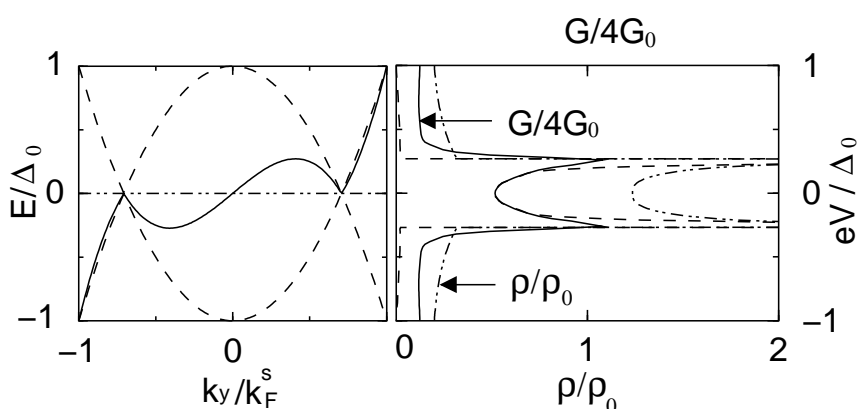

FIG. 4. The same curves as in Fig. 3 for the $f$-wave pairing potential (15) with vertical lines of nodes. 


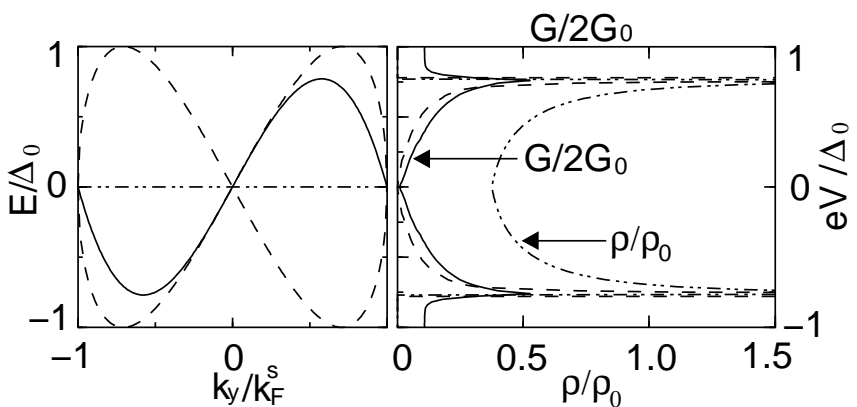

FIG. 5. The same curves as in Fig. 3 for the $f$-wave pairing potential 17 with vertical lines of nodes.

in the tunneling conductance and DOS curves occur at $e V= \pm 0.76 \Delta_{0}$. Their position is approximately the same as in Fig. 3 for the anisotropic $p$-wave. However, in Fig. 5, unlike in all other figures, tunneling conductance vanishes at zero bias, even though DOS remains finite. This characteristic feature of the $f$-wave potential (17) is a consequence of the square-root factor in Eq. (9).

Thus, tunneling conductance $\bar{G}(V)$ exhibits two peaks located symmetrically around $V=0$ for all pairing potentials with vertical lines of nodes described in this subsection. This conclusion is in agreement with recent numerical calculations 33.

\section{Pairing potentials with horizontal lines of nodes}

Thus far we considered pairing potentials that do not depend on $k_{z}$, the momentum component perpendicular to the $\mathrm{Sr}_{2} \mathrm{RuO}_{4}$ planes. Ref. [17] also proposed the $p$ wave with horizontal lines of nodes at certain $k_{z}$. Its pairing potential can be obtained from Eq. (10) by the following substitution

$$
\Delta_{0} \rightarrow \tilde{\Delta}_{0}\left(p_{z}\right)=\Delta_{0} \cos \left(p_{z}\right)
$$

where we switched to the dimensionless variable $p_{z}=$ $k_{z} c / \hbar$ ( $c$ is the interplane distance).

Now DOS is obtained by averaging $\rho(\epsilon)$ from Eq. (11) over $p_{z}$ using Eq. (19):

$$
\begin{aligned}
\rho(\epsilon) & =\int_{\left|\tilde{\Delta}_{0}\left(p_{z}\right)\right|>|\epsilon|} \frac{d p_{z}}{2 \pi} \frac{k_{F}}{h\left|\tilde{\Delta}_{0}\left(p_{z}\right)\right|} \\
& =\frac{\rho_{0}}{\pi} \ln \frac{1+\sqrt{1-\left(\epsilon / \Delta_{0}\right)^{2}}}{1-\sqrt{1-\left(\epsilon / \Delta_{0}\right)^{2}}} .
\end{aligned}
$$

In Eq. 20), $\tilde{\Delta}_{0}\left(p_{z}\right)$ vanishes linearly with $p_{z}$ at the nodes, which results in a logarithmic divergence of $\rho(\epsilon)$ at $\epsilon \rightarrow 0$ :

$$
\rho(\epsilon) \propto \ln \left|\Delta_{0} / \epsilon\right|, \quad \text { for } \quad|\epsilon| \ll \Delta_{0} \text {. }
$$

The logarithmic divergence is cut off at $\left|\tilde{\Delta}_{0}\left(p_{z}\right)\right|=|\epsilon|$, because Eq. (11) applies only to the subgap states with $|\epsilon|<\left|\tilde{\Delta}_{0}\left(p_{z}\right)\right|$. The plot of $\rho(\epsilon)$ is shown in Fig. 2 .
Tunneling conductance $\overline{\mathcal{G}}(V)$ is obtained by substituting Eq. (19) into Eq. (12) and averaging over $p_{z}$ :

$$
\begin{aligned}
\overline{\mathcal{G}}(V) & =2 G_{0} \int_{\left|\tilde{\Delta}_{0}\left(p_{z}\right)\right|>|e V|} \frac{d p_{z}}{2 \pi}\left(1-\left|\frac{e V}{\tilde{\Delta}_{0}\left(p_{z}\right)}\right|^{2}\right) \\
& =\frac{4}{\pi} G_{0}\left(\arccos \left|\frac{e V}{\Delta_{0}}\right|-\left|\frac{e V}{\Delta_{0}}\right| \sqrt{1-\left|\frac{e V}{\Delta_{0}}\right|^{2}}\right) .
\end{aligned}
$$

In Eq. 23), the divergence of $1 /\left|\tilde{\Delta}_{0}\left(p_{z}\right)\right|^{2}$ at the node is cut off at $|e V|$, because Eq. (12) applies only for $|e V|<$ $\left|\tilde{\Delta}_{0}\left(p_{z}\right)\right|$. To treat the divergence, it is convenient to change the variable of integration from $p_{z}$ to $\Delta$ :

$$
\overline{\mathcal{G}}(V)=2 G_{0}\left(1-\frac{4}{2 \pi} \int_{0}^{|e V|} \frac{d \Delta}{\Delta^{\prime}}-\frac{4|e V|^{2}}{2 \pi} \int_{|e V|}^{\Delta_{0}} \frac{d \Delta}{\Delta^{\prime} \Delta^{2}}\right)
$$

Here the factors of 4 come from the two nodal lines and the integration over the two sides of each line. The slope $\Delta^{\prime}=\left|d \tilde{\Delta}_{0}\left(p_{z}\right) / d p_{z}\right|$ is approximately constant at the nodes: $\Delta^{\prime} \approx \Delta_{0}$. Then the integrals in Eq. (25) can be taken, and we find

$$
\overline{\mathcal{G}}(V) \approx 2 G_{0}\left(1-\frac{4}{\pi}\left|\frac{e V}{\Delta_{0}}\right|\right) \quad \text { for } \quad|e V| \ll \Delta_{0} .
$$

Thus, tunneling conductance has a cusp at zero bias. The curve for $\bar{G}(V)$ calculated exactly starting from Eqs. (1) and (2) is shown in Fig. 2 and indeed demonstrates the cusp in agreement with Eq. (26).

Notice the difference in Fig. 2 between the singular logarithmical divergence of DOS and the triangular-shaped cusp in tunneling conductance at zero bias. Both features result from the pile-up of surface Andreev states at zero energy caused by vanishing gap at the nodes. These are robust features of the chiral pairing potentials with horizontal lines of nodes, independent of the band structure details. While Eqs. (21) and (24) are derived specifically for the gap 19) being a cosine function, the asymptotic Eqs. 22) and (26) are valid for any generic chiral pairing potential with horizontal lines of nodes. For example, the $f$-wave with horizontal lines of nodes [19],

$$
\begin{aligned}
\Delta_{6}(\mathbf{k}) & =i \Delta_{0} \sin \left(p_{z}\right)\left(k_{x}+i k_{y}\right)^{2} /\left(k_{F}^{(s)}\right)^{2}, \\
E_{6}\left(k_{y}\right) & =\operatorname{sgn}\left(k_{y}\right) \Delta_{0}\left[2\left(k_{y} / k_{F}^{(s)}\right)^{2}-1\right]\left|\sin \left(p_{z}\right)\right|,
\end{aligned}
$$

produces similar curves for the in-plane tunneling. However, being an odd function of $p_{z}$, it also exhibits a ZBCP in the $c$-axis tunneling.

\section{COMPARISON WITH EXPERIMENT}

Point-contact tunneling spectroscopy 29 found a $\mathrm{ZBCP}$ in $\mathrm{Sr}_{2} \mathrm{RuO}_{4}$. The data were fitted using the $2 \mathrm{D}$ 


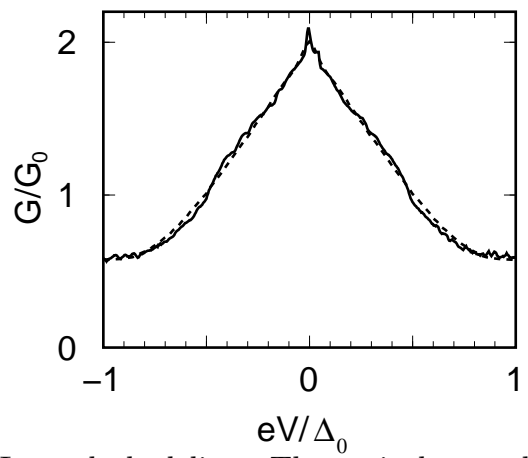

FIG. 6. Long-dashed line: Theoretical tunneling conductance curve from our Fig. 2 calculated for the $p$-wave with horizontal lines of nodes [Eq. (19)] using $\Delta_{0}=0.8 \mathrm{meV}$. Solid line: Experimental curve from Fig. 2 of Ref. 30] for $0.8 \mathrm{~K}$.

isotropic $p$-wave (10) and assuming a narrow acceptance cone, i.e. a small value of $k_{y}^{\max }$. In this situation, only the states with $k_{y} \approx 0$ and hence $E\left(k_{y}\right) \approx 0$ are probed; thus all pairing potentials, except the $f$-wave (17), would show a ZBCP. However, such a ZBCP, in contrast to nonchiral cuprates [25] and organic superconductors 34, does not identify the pairing symmetry uniquely.

The more recent tunneling experiments [30] were performed on cleaved junctions of $\mathrm{Sr}_{2} \mathrm{RuO}_{4}$ with inclusions of $\mathrm{Ru}$. Although nominally their setup corresponds to tunneling along the $c$ axis, the experimentalists believe that the actual tunneling takes place in the $(a, b)$ plane via the inclusions of Ru. They found a cusp-like peak at zero bias in the $1.4-\mathrm{K}$ phase and a sharper ZBCP in the 3 -K phase. The latter was attributed to a nonchiral superconductivity developing at the grain boundaries [35]. An experimental curve from Ref. [30] representing the 1.4-K phase is shown in Fig. 6. It has the characteristic triangular shape with a cusp at zero bias and agrees very well with our theoretical curve calculated for the pairing potential (19). To making the comparison, we treated the horizontal and vertical scales and the vertical offset (the background) of the theoretical curve as fitting parameters. The best fit corresponds to the gap $\Delta_{0}=0.8$ meV.

The good agreement between the theoretical and experimental curves is a strong indication in favor of the $p$ or $f$-wave pairing potential with horizontal lines of nodes in the 1.4-K phase. Further measurements showing the absence or presence of a ZBCP in the $c$-axis tunneling could discriminate between the $p$ - and $f$-wave cases. Experiment 30] found that an additional sharp ZBCP with the width of the order of $0.1 \mathrm{meV}$ develops on top of the cusp at lower temperatures 0.5 and $0.32 \mathrm{~K}$. It may be attributed to a contribution from the $c$-axis tunneling, if the pairing potential is the $f$-wave (27).

The described above scenario corresponds to the horizontal nodes in the main electron band $\gamma$ of $\mathrm{Sr}_{2} \mathrm{RuO}_{4}$. In the alternative scenario proposed in Ref. [21], the main band $\gamma$ is nodeless, but the two other bands $\alpha$ and $\beta$ have horizontal lines of nodes. In this case, one would expect a superposition of a parabolic curve for the nodeless band and triangular curves for the bands with nodes. Perhaps the sharp ZBCP developing at low temperatures 0.5 and $0.32 \mathrm{~K}$ could be interpreted as the triangular curve corresponding to the gap of the order of $0.1 \mathrm{meV}$ in the $\alpha$ and $\beta$ bands.

\section{CONCLUSIONS}

We derived the analytical formula (9) for subgap tunneling at low transparency of the barrier, which takes into account only the contribution of the surface Andreev states. This formula produces tunneling curves in a simple and physically transparent way. For the chiral pairing potentials with vertical lines of nodes, tunneling curves show double peaks, which originate from the turning points in the energy dispersion of the surface Andreev states. On the other hand, for the chiral pairing potentials with horizontal lines of nodes, we find a single triangular-shaped peak with a cusp at zero bias, which results from the pile-up of the surface Andreev state at zero energy caused by vanishing gap at the nodes.

Double peaks in tunneling conductance were observed experimentally in some point contacts with $\mathrm{Sr}_{2} \mathrm{RuO}_{4}$ in Ref. [29]. However, such double-peaked spectra were associated with contacts with high transparency of the barrier [29], a regime that is not addressed in our paper. On the other hand, no double peaks were observed in experiment [30], which only found a single triangularshaped peak at zero bias. As shown in Fig. 6, this peak is very well fitted by our calculations for horizontal lines of nodes. It is also visually similar to the single peak found for contacts with low transparency in Fig. 3 of experiment 229] (which shows resistance, rather than conductance). Thus, we conclude that the superconducting pairing potential in $\mathrm{Sr}_{2} \mathrm{RuO}_{4}$ most likely has horizontal lines of nodes.

Note added in proof. Quantitative comparison shows that our calculation for horizontal lines of nodes also fits the experimental data of Ref. 29 very well. The curves are shown at http://www2.physics.umd.edu/ yakovenk/talks/Sr2RuO4

We are grateful to Ying Liu and Gernot Goll for sending us the experimental data of Refs. 30 and 29, and to Yoshiteru Maeno, Ying Liu, and Igor Z̆utić for useful discussions. This work was supported by the Packard Foundation and by the NSF Grant DMR-9815094. 
[1] Y. Maeno, H. Hashimoto, K. Yoshida, S. Nishizaki, T. Fujita, J. G. Bednorz, and F. Lichtenberg, Nature (London) 372, 532 (1994).

[2] Y. Maeno, T. M. Rice, and M. Sigrist, Physics Today 54, \#1, 42 (January 2001); 54, \#3, 104 (March 2001).

[3] A. P. Mackenzie, R. K. W. Haselwimmer, A. W. Tyler, G. G. Lonzarich, Y. Mori, S. Nishizaki, and Y. Maeno, Phys. Rev. Lett. 80, 161 (1998); Z. Q. Mao, Y. Mori, and Y. Maeno, Phys. Rev. B 60, 610 (1999).

[4] K. Ishida, Y. Kitaoka, K. Asayama, S. Ikeda, S. Nishizaki, Y. Maeno, K. Yoshida, and T. Fujita, Phys. Rev. B 56, R505 (1997).

[5] K. Ishida, H. Mukuda, Y. Kitaoka, K. Asayama, Z. Q. Mao, Y. Mori, and Y. Maeno, Nature (London) 396, 658 (1998); K. Ishida, H. Mukuda, Y. Kitaoka, Z. Q. Mao, H. Fukazawa, and Y. Maeno, Phys. Rev. B 63, 060507(R) (2001).

[6] G. M. Luke, Y. Fudamoto, K. M. Kojlma, M. I. Larkin, J. Merrin, B. Nachumi, Y. J. Uemura, Y. Maeno, Z. Q. Mao, Y. Mori, H. Nakamura, and M. Sigrist, Nature (London) 394, 558 (1998).

[7] T. M. Riseman, P. G. Kealey, E. M. Forgan, A. P. Mackenzie, L. M. Galvin, A. W. Tyler, S. L. Lee, C. Ager, D. McK. Paul, C. M. Aegerter, R. Cubitt, Z. Q. Mao, T. Akima, and Y. Maeno, Nature (London) 396, 242 (1998); P. G. Kealey, T. M. Riseman, E. M. Forgan, L. M. Galvin, A. P. Mackenzie, S. L. Lee, D. McK. Paul, R. Cubitt, D. F. Agterberg, R. Heeb, Z. Q. Mao, and Y. Maeno, Phys. Rev. Lett. 84, 6094 (2000).

[8] T. M. Rice and M. Sigrist, J. Phys. Condens. Matter 7, L643 (1995); M. Sigrist, D. Agterberg, A. Furusaki, C. Honerkamp, K. K. Ng, T. M. Rice, and M. E. Zhitomirsky, Physica C 317-318, 134 (1999).

[9] S. NishiZaki, Y. Maeno, and Z. Mao, J. Phys. Soc. Jpn. 69, 572 (2000).

[10] K. Ishida, H. Mukuda, Y. Kitaoka, Z. Q. Mao, Y. Mori, and Y. Maeno, Phys. Rev. Lett. 84, 5387 (2000).

[11] I. Bonalde, B. D. Yanoff, M. B. Salamon, D. J. Van Harlingen, E. M. E. Chia, Z. Q. Mao, and Y. Maeno, Phys. Rev. Lett. 85, 4775 (2000).

[12] M. A. Tanatar, S. Nagai, Z. Q. Mao, Y. Maeno, and T. Ishiguro, Physica C 341-348, 1841 (2000); Phys. Rev. B 63, 064505 (2001).

[13] H. Matsui, Y. Yoshida, A. Mukai, R. Settai, Y. Onuki, H. Takei, N. Kimura, H. Aoki, and N. Toyota, Phys. Rev. B 63, 060505(R) (2001).

[14] C. Lupien, W. A. MacFarlane, C. Proust, L. Taillefer, Z. Q. Mao, and Y. Maeno, Phys. Rev. Lett. 86, 5986 (2001).

[15] Vertical and horizontal lines of nodes were discussed for cuprate superconductors by R. J. Radtke, A. I. Liechtenstein, V. M. Yakovenko, and S. Das Sarma, Phys. Rev. B 53, 5137 (1996).

[16] K. Miyake and O. Narikiyo, Phys. Rev. Lett. 83, 1423 (1999).

[17] Y. Hasegawa, K. Machida, and M. Ozaki, J. Phys. Soc. Jpn. 69, 336 (2000).

[18] M. J. Graf and A. V. Balatsky, Phys. Rev. B 62, 9697 (2000).

[19] H. Won and K. Maki, Europhys. Lett. 52, 427 (2000).

[20] D. F. Agterberg, T. M. Rice, and M. Sigrist, Phys. Rev. Lett. 78, 3374 (1997).
[21] M. E. Zhitomirsky and T. M. Rice, Phys. Rev. Lett. 87, 057001 (2001).

[22] M. A. Tanatar, M. Suzuki, S. Nagai, Z. Q. Mao, Y. Maeno, and T. Ishiguro, Phys. Rev. Lett. 86, 2649 (2001); K. Izawa, H. Takahashi, H. Yamaguchi, Y. Matsuda, M. Suzuki, T. Sasaki, T. Fukase, Y. Yoshida, R. Settai, and Y. Onuki, ibid. 86, 2653 (2001).

[23] T. Dahm, H. Won, and K. Maki, cond-mat/0006301.

[24] S. Kashiwaya, Y. Tanaka, M. Koyanagi, H. Takashima, and K. Kajimura, Phys. Rev. B 51, 1350 (1995); M. Covington, M. Aprili, E. Paraoanu, L. H. Greene, F. Xu, J. Zhu, and C. A. Mirkin, Phys. Rev. Lett. 79, 277 (1997); J. Y. T. Wei, N.-C. Yeh, D. F. Garrigus, and M. Strasik, ibid. 81, 2542 (1998).

[25] C.-R. Hu, Phys. Rev. Lett. 72, 1526 (1994); J. Yang and C.-R. Hu, Phys. Rev. B 50, 16766 (1994).

[26] L. J. Buchholtz and G. Zwicknagl, Phys. Rev. B 23, 5788 (1981).

[27] M. Yamashiro, Y. Tanaka, and S. Kashiwaya, Phys. Rev. B 56, 7847 (1997); M. Yamashiro, Y. Tanaka, Y. Tanuma, and S. Kashiwaya, J. Phys. Soc. Jpn. 67, 3224 (1998); N. Yoshida, Y. Tanaka, J. Inoue, and S. Kashiwaya, ibid. 68, 1071 (1999); M. Yamashiro, Y. Tanaka, N. Yoshida, and S. Kashiwaya, ibid. 68, 2019 (1999).

[28] C. Honerkamp and M. Sigrist, J. Low T. Phys. 111, 895 (1998).

[29] F. Laube, G. Goll, H. v. Löhneysen, M. Fogelström, and F. Lichtenberg, Phys. Rev. Lett. 84, 1595 (2000).

[30] Z. Q. Mao, K. D. Nelson, R. Jin, Y. Liu, and Y. Maeno, Phys. Rev. Lett. 87, 037003 (2001).

[31] G. E. Blonder, M. Tinkham, and T. M. Klapwijk, Phys. Rev. B 25, 4515 (1982).

[32] Y. Tanaka and S. Kashiwaya, Phys. Rev. Lett. 74, 3451 (1995).

[33] N. Stefanakis, J. Phys. Condens. Matter 13, 3643 (2001); cond-mat/0109498.

[34] K. Sengupta, I. Zutić, H.-J. Kwon, V. M. Yakovenko, and S. Das Sarma, Phys. Rev. B 63, 144531 (2001).

[35] M. Sigrist and H. Monien, J. Phys. Soc. Jpn. 70, 2409 (2001). 